\title{
Integrating Delphi Consensus Consultation and Community- Based Participatory Research
}

\author{
Melinda J. Suto, Sara Lapsley, Anusha Balram, Steven J. Barnes, Sharon Hou, Dragos \\ C. Ragazan, Jehannine Austin, Mike Scott, Lesley Berk, Erin E. Michalak
}

\begin{abstract}
Delphi consensus consultation methods and community-based participatory research (CBPR) are distinct approaches that have traditionally been employed separately. This paper explores the integration of Delphi methods with CBPR in a research project that sought to identify effective self-management strategies for bipolar disorder (BD). We introduce our Canadian-based network which specializes in CBPR in BD, and outline the key principles of CBPR approaches. Delphi consensus consultation methods are described and we present the five phases of our Delphi consensus consultation project, conducted within a CBPR framework. Examples of how each project phase incorporated the principles of CBPR are provided, as are personal reflections of community members involved in the project, and broader reflections on challenges commonly encountered in CBPR projects.
\end{abstract}

KEYWORDS Delphi consensus consultation, community-based participatory research, bipolar disorder, self-management

Bipolar disorder (BD) is a condition characterized by pronounced variability in mood, activity, and energy levels, with mood episodes ranging from periods of clinical depression through to mild elation ('hypomania'), or extreme elation and/or irritability ('mania') (Goodwin \& Redfield-Jamison, 2007). These extremes of mood can have a negative effect on activities of daily living such as employment, education, relationships, and on other domains that contribute to quality of life (QoL) (Michalak, Yatham, Kolesar, \& Lam, 2006; Rosa et al., 2010). As robust epidemiological studies indicate a 1-2\% lifetime prevalence, around 500,000 Canadians live with the condition (Merikangas et al., 2007) correlates, and treatment patterns of bipolar spectrum disorder in the US population. DESIGN: Direct interviews. SETTING: Households in the continental United States. PARTICIPANTS: A nationally representative sample of 9282 English-speaking adults (aged > or=18 years. Bipolar disorder research has burgeoned over the past two decades with much of it conducted in the biomedical realm, examining the biological causes and consequences of the condition and pharmacological treatment approaches. Although pharmacology is typically the bedrock of treatment in $\mathrm{BD}$, pharmacological interventions alone are usually insufficient to ensure optimal health and QoL; psychosocial treatments and factors also impact patient outcomes (Chatterton et 
al., 2017)and conventional meta-analyses provided limited comparisons between therapies. Aims To combine evidence for the efficacy of psychosocial interventions used as adjunctive treatment of bipolar disorder in adults, using network meta-analysis (NMA. Our Canadian network has been advancing research into psychosocial factors in BD, particularly in selfcare or 'self-management' approaches to the condition. In this paper, we describe a project which synergistically combined two traditionally distinct research approaches-communitybased participatory research (CBPR) and Delphi consensus consultation-in order to build knowledge on effective self-management strategies for BD.

\section{Introduction to CREST.BD}

The Collaborative RESearch Team to study psychosocial issues in Bipolar Disorder (CREST. BD), established in 2007, is a multidisciplinary network committed to creating and sharing knowledge that advances research into the psychosocial facets of BD (Michalak et al., 2012; Michalak et al., 2015). CREST.BD specializes in the application of CBPR in BD research and knowledge exchange. The values articulated in CREST.BD's strategic vision are particularly relevant to CBPR and include: wellness and resilience, which is evident in our strengths-oriented approach; equity, which directs us to conduct research to address the social injustices seen from the often inequitable access to healthcare services by marginalized groups; and diversity, which manifests as our search for different opinions, and respect for various types of expertise (Michalak et al., 2016a). Furthermore, CREST.BD defines evidence and expertise broadly, and thus regards the contributions of peer-reviewed scientific findings, the views of people with lived experience of $\mathrm{BD}$, and clinical expertise as all equally credible and necessary to advance knowledge about BD (Sackett, Straus, Richardson, Rosenberg, \& Haynes, 2000).

\section{Defining CBPR}

CBPR first arose from the movements led by educator-activist Paulo Freire that sought to emancipate and empower illiterate and marginalized Brazilian communities (Freire, 1972). It is an action-oriented research approach underpinned by critical social theories, which question the taken-for-granted assumptions about what is truly normal and what is instead socially constructed. CBPR involves the co-construction of knowledge through the open dialogue between various partners in order to raise awareness and to think critically about a given issue (i.e., conscientization). Attention is also paid to the relations between stakeholders, and efforts are made to reduce inequalities and power asymmetries, and to resolve other identified issues. Beginning in the late 1990s, North American health researchers further advanced the concept of CBPR by outlining eight key principles to guide the broader research community in using CBPR as an agent of social action and change (Israel, Schulz, Parker, \& Becker, 1998; Minkler \& Wallerstein, 2003). These principles, as first presented by Israel et al. (1998), are listed below and four are highlighted for their particular relevance to the study described in this paper:

\section{Recognizes community as a unit of identity}

\section{Builds on strengths and resources within the community}




\section{Facilitates collaborative partnership in all phases of the research \\ 4. Integrates knowledge and action for mutual benefit of all partners \\ 5. Promotes a co-learning and empowering process that attends to social inequalities \\ 6. Involves a cyclical and iterative process \\ 7. Addresses health from both positive and ecological perspectives \\ 8. Disseminates findings and knowledge gained to all partners. (pp. 178-180)}

The first principle recognizes that community extends beyond given geographical boundaries and includes people who share particular identities or social roles and therefore, who may experience similar experiences or fates associated with such (Israel, Eng, Schulz, Parker, \& Satcher, 2005; Viswanathan et al., 2004). The BD community is diverse and includes multiple stakeholders. This inclusivity attracts multiple voices, with varied perspectives, which are essential for the creation of collaborative and authentic partnerships; another noteworthy CBPR principle. These effective partnerships in turn: a) demonstrate communication that is both transparent and open to external information, b) foster decision-making that addresses power imbalances between groups and/or individuals, and c) establish a willingness to negotiate project goals. One way to redistribute power is to conceptualize, and to work with community partners as co-researchers instead of as 'subjects' of research. Historically, it was rare to design and conduct BD research that used a CBPR approach, wherein people with lived experience of the condition engage as co-creators of knowledge. Notably, CREST.BD has incorporated this innovation into multiple projects, described fully elsewhere (Michalak, et al., 2015). This change positions co-researchers as people with considerable agency who are then expected to be involved in the decisions that affect their communities. The traditional power imbalance between academia and the community is further disrupted as the community gives input on what is important to study, identifying who should be involved, and determining relevant knowledge exchange strategies.

Another CBPR principle is to acknowledge the strengths and resources of each partner and to further develop these over time (Israel et al., 2005). For example, non-profit mental health partners excel in networking, service provision, and advocacy, whereas academic partners have research skills and experience, dedicated time for research, and different funding opportunities. Finally, CBPR cultivates the practice of co-learning, and increases the capacity of all research partners. This principle is enacted by sharing diverse skills and resources, and by the expectation that knowledge does develop through an understanding of each other's perspectives and experiences. For example, CREST.BD initiated a Community Advisory Group (CAG) in 2009 to receive guidance and input for then current and future research directions. The CAG includes people with lived experience of $\mathrm{BD}$, mental health community and/or consumer organization representatives and healthcare providers. This advisory group exemplifies CBPR principles as it sustains a partnership wherein members can draw on each other's skills and share resources, and also build capacity in the broader BD community by optimizing networking opportunities, and providing a mechanism for co-learning. Among 
the specific objectives of the CAG are to: 1) be a resource for CREST.BD by facilitating the planning, implementation, evaluation, and distribution of research and knowledge exchange and, 2) identify barriers to the network's research and knowledge exchange initiatives, and help problem-solve related solutions.

Substantial and sustained community engagement is essential for CBPR and includes: the identification of specific health issue(s) or concern(s); the development of comprehensive action plans consisting of research question(s), goal(s), and method(s) (e.g., data collection and analyses); and the dissemination of findings (Schneider, 2012). Ideally, community partners are involved in each phase of the research process; however, this involvement can vary with each project, and with the available resources and time that partners have. This level of community engagement reflects working with communities instead of merely locating research opportunities within communities of interest.

It is common for CBPR to incorporate diverse methods (e.g., flexible use of quantitative, qualitative, arts-based, and mixed-method designs) to meet study objectives. However, one opportunity that has been under-explored and exploited is the combination of CBPR with the Delphi consensus consultation method, described below.

\section{Overview of the Delphi Consensus Consultation Method}

In ancient Greece, people would consult the Delphi oracle to gain information about future events so they could have an advantage when making difficult, albeit important decisions (Ilieva, 2013). The RAND Corporation created the Delphi technique in the 1950s for the purpose of forecasting and developing prediction ability to apply in various arenas e.g., social and political. Early developers rejected the customary use of in-person consensus meetings, with their potential of unwanted persuasion and influence between participants (Gordon \& Helmer 1964). Instead, panels of experts completed written questionnaires (called "sequential individual interrogations") and researchers incorporated participants' rationales from earlier responses into subsequent questions (Gordon \& Helmer, 1964, p. 5). Further development of Delphis aimed for 'stability in responses' over consensus and the use of data to inform policy and decision-making (Linstone \& Turoff, 2011). The Delphi consensus consultation method has evolved into a highly useful and structured approach to address complex problems (Davidson, 2013). It is particularly effective when there is scant scientific evidence available to guide problem-solving, or when existing evidence needs to be clarified, improved or translated into everyday practice (Minas \& Jorm, 2010; Vázquez-Ramos, Leahy, \& Estrada Hernández, 2007).

Rowe and Wright's (1999) Delphi systematic review concluded by recognizing the ongoing development and application of this technique. Recent innovations have included a hybrid Delphi that utilizes the best features of other approaches e.g., focus groups, classic Delphis, nominal group techniques (Landeta, Barrutia \& Lertxundi, 2011). Scholars working from the epistemological position that underpins the participatory paradigm have developed and used Delphi methods in ways that put their principles into practice (Kezar \& Maxey, 2016). Their 'change-oriented' Delphi offered an incisive examination of this compatibility with six common

Engaged Scholar Journal: Community-Engaged Research, Teaching, and Learning 
features of participatory studies that are well-aligned with those described in this paper. One example of another Delphi innovation was Totikidis' (2010) use of a nominal group technique, which had participants generating ideas for an intervention to improve community health and rank-ordering these for future implementation. Fletcher and Marchildon's (2014) two-round 'modified Delphi' used interviews and then questionnaires derived from inductive analysis for the purpose of program evaluation at a health systems level. These examples highlight some of the Delphi approaches that have been used within a participatory paradigm, however, the integration of CBPR as articulated in this paper and Delphi consensus consultation approaches has seldom been explored specifically within mental health research.

At the core of the Delphi consensus consultation method is a number of experts who contribute their independent views and ratings in an iterative process (e.g., survey rounds) insofar as substantial consensus can be achieved (Amos \& Pearse, 2008; Jorm, 2015; Powell, 2003). Although there are many types of Delphi approaches to select from, we chose the method used by Jorm (2015), the steps of which are summarized here. The first step is the formulation of the research question(s). Literature reviews, and other sources of information, for example, from focus group discussions or from meetings with researchers tend to facilitate this step (Amos \& Pearse, 2008; Vernon, 2009). Step two involves the selection of the expert panel, which Jorm (2015) encourages to be based on Suroweiki's (2004) four guidelines of diversity, anonymity, autonomy, and aggregation. Experts are clearly defined and may include professionals, persons with lived experience of the given issue(s), and other related stakeholders. Step three determines the panel size, which requires recognition that larger panels may indeed reduce the overall influence of an individual and therefore increase the stability of opinions. Step four uses academic and grey literature to help develop the surveys. Step five involves providing panelists with any additional information that may assist with their responses (e.g., survey objectives, instructions). Steps six and seven include survey administration, and analysis of responses so feedback could be provided to panelists for any subsequent survey rounds. For example, panelists may receive the percentage of the group's agreement on particular survey items that did not quite reach the required level of consensus, and in comparison, with their own ratings (Berk, Jorm, Kelly, Dodd, \& Berk, 2011). This then allows panelists to change or maintain their ratings on these items anonymously (Donohoe, Stellefson, \& Tennant, 2012). The final step involves reporting the Delphi survey results, where a variety of methods may be used.

The use of online surveys makes accessing large and diverse international samples feasible given this medium is both cost and time efficient (Donohoe et al., 2012). Furthermore, participants can rate survey items in private and when convenient; hence encouraging a freer expression of opinions. A strength of the Delphi method is in its flexible application to many areas of research, including health, and more recently, in BD (Berk et al., 2011; Nair, Aggarwal, \& Khanna, 2011; Vernon, 2009). There is also clear compatibility between the Delphi methods and CBPR approaches. For example, both value various types of expertise (e.g., lived experience, clinical, and academic expertise). 


\section{A CBPR and Delphi Community Consultation Study on Bipolar Disorder Self- Management Strategies}

The research question that this CBPR-Delphi consensus consultation study addressed was: What self-management strategies (SMSs) do both people with BD and BD healthcare providers deem most effective for: 1) maintaining balance in mood, and 2) stopping progression into hypomania and mania? Here we offer a brief overview of the study in order to provide a context for the subsequent examination of the Delphi team and working relationships within it. A full report of the primary study findings appears in Michalak et al. (2016b). The inclusion criteria for participants with lived experience of BD were: 19 years and older; an ability to communicate in English; and a self-reported diagnosis of BD1, BD1 or NOS. For the healthcare provider participants the inclusion criteria were: 19 years and older; an ability to communicate in English; and self-reported work with individuals with BD. If participants fit both inclusion criteria ( $\mathrm{n}=3$ ), they selected their panel. In Round 1, 101 participants with $\mathrm{BD}$ and 52 healthcare providers completed a 493-item survey using a Likert-type rating scale. In Round 2, $83(82 \%)$ and $43(83 \%)$ participants, respectively, completed a similar survey with 155 items.

It was found that both panels of experts, people with BD and healthcare providers, generally agree on the same categories of SMSs to maintain balance and to limit the onset of hypomania and mania. To best maintain balance, both panels identified strategies that relate to medication and stress management, and that ensure adequate sleep, rest and exercise as key. Similarly, both panels found strategies that recognize early-warning symptoms, and that promote sleep, rest, and medication management to be effective in the prevention of elevated mood states. Analysis of the study data yielded by exploratory factor analysis pointed to some underlying factors that may connect preferred SMSs. In regard to maintaining a balanced mood, factors of calming oneself, medical management, maintaining hope, and physical activity were key. For stopping the progression into hypomania and mania, strategies connected by factors of planning ahead, intervening early, and decreasing the use of stimulants were apparent.

\section{Delphi Consensus Consultation Team and Working Relationships}

The Delphi research team was comprised of two peer researchers (i.e., people with lived experience of BD who were not academics), four undergraduate student volunteers, two research coordinators, a knowledge translation specialist, and four academic researchers. The diversity of the team, and its inclusive research tasks encouraged putting CBPR principles into practice. The following description presents the five core Delphi project phases, and provides examples of how the eight aforementioned CBPR principles were enacted during these phases.

Phase one involved the re-analysis of a qualitative research dataset (see Suto, Murray, Hale, Amari, \& Michalak, 2010), which included transcripts from interviews and focus groups in order to extract candidate SMSs. Peer researcher Lapsley completed this analysis, with academic team members providing ongoing mentorship. Also, during this phase, peer researcher Scott produced a project definition of self-management - which can encompass a wide range of plans, activities or routines, such as monitoring mood, education, optimizing diet, exercise and 
sleep, pursuing creative activities, or engaging in meditation and relaxation activities - which was refined by group consensus. The definition (Michalak et al., 2016b) reads:

Bipolar disorder (BD) self-management refers to the plans and/or routines that a person with $\mathrm{BD}$ uses to promote health and QoL. Healthcare professionals can provide information about BD self-management strategies and support for their application. Family, friends and caregivers can also be involved in developing strategies and supporting the person in using them. However, outside of hospital settings, it is typically the person with $\mathrm{BD}$ who chooses their approach to self-management and enacts and tailors their own strategies. Most strategies, regardless of whether they originate solely with the person with BD or are developed in collaboration with others, can be considered self-management strategies). (p. 81)

Phase one benefitted from the second CBPR principle of building on the community's existing strengths and resources, including the lived experience and expertise of the team's peer researchers. The frequent collaborative team meetings, ongoing peer researcher support and guidance, and the overall spirit of collegiality reflected the fifth CBPR principle of promoting co-learning and capacity building. The academic researchers learned about the Delphi methods in tandem with the other team members, and encouraged shared decisionmaking within the group to foster a dynamic and inclusive learning environment. The team's definition and selection of SMSs echoed the seventh CBPR principle, in recognizing the importance of the local relevance of bipolar self-management, and understanding that there are multiple predictors of health and well-being.

In phase two, the peer researchers reviewed and evaluated grey literature sources to identify additional potential self-management strategies. This activity required intensive combing of websites and other online resources, such as podcasts, blogs, and reports. Throughout this process, two academic researchers provided mentoring and guidance to the peer researchers, as did a research coordinator, who also examined the peer-reviewed literature. Throughout these activities, there were frequent team meetings, where decentralized decision-making was encouraged, reflecting the third CBPR principle of collaborative partnerships and shared power processes.

In phase three, all the previously identified and aggregated SMSs were subjected to content analysis that accommodates inductive and deductive approaches for qualitative and quantitative data (Elo \& Kyngäs, 2008). Our process involved assigning each SMS into categories, using some from a previous study (e.g., connecting with others) and naming others through consensus. Next, we reduced the number of strategies by eliminating duplicates (3598) or unintelligible text (111). Then we clarified some of the wording to create understandable, actionable items for the survey. This process produced 493 SMSs to be selected for the development of the online surveys, which were to be disseminated to the two expert panels: people with lived experience of $\mathrm{BD}$, and $\mathrm{BD}$ healthcare providers. During this stage, the entire team spent several months organizing the candidate SMSs. These tasks required all team 
members to voice their different perspectives, and accordingly these discussions strengthened analysis and decision-making. As the Delphi method was new to all team members, these synergistic and collective dialogues exemplified the fifth CBPR principle of co-learning and capacity building. Overall, the work in phase three was an iterative cyclical process, as per the sixth CBPR principle.

In phase four, the panel experts were recruited and the Delphi consensus consultation was launched as a two-round online survey. Potential participants were recruited through an electronic invitation and accompanying consent form that was sent to a mailing list of approximately 500 people (350 individuals who are on the CRESTBD email list, and 150 individuals who were selected by the co-researchers from their academic and professional networks). These recruitment materials were circulated to prominent mental health and research organizations as well. Researchers also developed and circulated two recruitment videos on the CREST.BD YouTube channel. These strategies offered the best chance of recruiting people with lived experience of $\mathrm{BD}$ and $\mathrm{BD}$ healthcare providers who could form the panel experts. The community advisory group was critical for boosting numbers of participants after the initial recruitment strategy occurred, and recommended strategies such as shareable presentations and video outputs that each member could further distribute to their networks. These strategies were then co-developed with people with lived experience to provide the broader community with an introduction to the survey, the rationale for it, and reflections on its expected impact. Blogs written in plain language by peer and academic researchers and webinars delivered by project academics and peer researchers intensified recruitment efforts and kept the larger BD community abreast of the project's progress. Overall, these efforts were consistent with the CBPR paradigm and relied on the strengths of partners with lived experience to recruit experts (i.e., second principle), to disseminate knowledge (i.e., eighth principle), and based on the dynamic dialogue with the BD community (i.e., sixth principle), to refine the survey as needed.

Participants used Likert-type scales to rate the perceived helpfulness of each of the 493 SMSs for maintaining a balanced mood and for stopping the onset of hypomania/mania. In regard to the survey design, a subset of the research team contributed to the initial draft of the survey items and peer researchers, in particular offered feedback so future revisions could be more clear and user-friendly, especially for people with lived experience. The decision to include both people with lived experience and healthcare providers established diversity in expert opinion, and therefore met existing Delphi guidelines (Jorm, 2015) and recognized the broader $\mathrm{BD}$ community as a unit of identity - the first CBPR principle. In turn, the team enacted the second CBPR principle of building on the strengths and resources of the community as it relied on the BD community's knowledge of self-management, and on the peer researchers' capacities to assist with the survey design.

In phase five, the academic researchers used exploratory factor analysis to identify the underlying factors that link effective SMSs, and the team as whole participated in knowledge translation activities to disseminate the top-rated SMSs to the broader BD community. In addition to traditional academic outputs, such as peer-reviewed publications, the top-rated 
SMSs identified were included on the CREST.BD 'Bipolar Wellness Centre' (www.bdwellness. com), an online resource where the community can access evidence-based tools and tailored information to learn more about $\mathrm{BD}$ and self-management to improve health and QoL. A balance between knowledge generation and action for benefit (i.e., the fourth CBPR principle) was therefore met. Given that people with lived experience were also essential for disseminating study progress and findings to the community (e.g., co-authorship of academic publications, blogging, social media posts), this phase was congruent with the eighth CBPR principle of involving partners in knowledge exchange.

Although establishing long-term relationships with the communities of interest is not an explicit CBPR principle, Israel et al. (1998) emphasize the importance and necessity of prolonged engagement and commitment. Throughout the project phases, the team drew upon and nurtured each other's skills and resources. The team's research capacity and knowledge of BD was also fostered; for instance, our student volunteers were trained in basic data analysis, received academic supervision, and participated in team meetings to advance their knowledge of $\mathrm{BD}$ and self-management, and gained exposure to CBPR. Capacity building occurred; one research coordinator later joined CREST.BD as a $\mathrm{PhD}$ graduate student, and one student volunteer was later appointed as a research assistant, and the team's peer researchers' personal and professional development was fostered. Peer researcher Lapsley reflected on her experiences:

My subjective experience as a peer researcher encompassed multiple identities. In some ways, identifying as a peer did not have a particular impact; the tasks allotted were the same as other team members who did not have lived experience, and the skills that I gained were invaluable in my role as a 'regular' researcher in other contexts. However, the role of the peer researcher was a privileged identity in that I was able to collaborate with some of the world's experts in BD; an opportunity I might not have had as a typical graduate student. Acting as a peer researcher helped me to acquire knowledge about the condition that I live with, and the sheer number of selfmanagement strategies that the Delphi team gathered was encouraging. Unfortunately, the research process was distressing at times. On internet forums, I observed people living with $\mathrm{BD}$ who were demonstrating acute mood states and symptoms such as hypersexuality. It was a stark reminder of how difficult it can be to live with BD, and brought back painful memories of previous episodes. Despite this, the personal connection that I felt with the topic of study made my work as a peer researcher a meaningful and empowering experience.

Peer researcher Scott also explained how his role influenced, and helped him with other aspects of his life:

Being involved in the CREST.BD Delphi study gave me the opportunity to make a difference. Not only have I had the chance to learn research skills, and pursue my goals of a career in mental health, but I have also been given the tremendous opportunity 
to help improve the lives of others. I have been able to take pride and build my selfesteem by giving back to the community. The research we have done has encouraged the development of new approaches for managing mental health, and BD specifically. This is so important. Also, along the way, I have had the opportunity to build my own knowledge of effective coping strategies. This has been an excellent benefit to working on the project. It is unlikely that the discovery of strategies such as the ones we identified would be recognized in a typical biomedical research lab. I see such powerful benefits to combining the CBPR and Delphi methodology. I notice three key advantages to this methodology. First, there is a potential boost to self-esteem for people living with the condition and participating in research. Second, peer researchers and community members that live with the condition have a naturally strong drive to help for causes in their own life, and this opens the doors to productivity in research. Finally, and perhaps most important in this research is that working with people who have the lived condition taps into first-hand insight and experience that can provide keys for developing new strategies for coping and wellness.

Overall, the Delphi project leaders fostered a co-learning environment, whereby team members worked freely and collaboratively without the inherent power asymmetries that are often found given the diverse educational attainments and roles (e.g., student volunteers, persons with lived experience of $\mathrm{BD}$, and academic researchers), and thus the process was consistent with CBPR practices.

\section{Challenges and Solutions to Enacting CBPR}

To present our aforementioned study with full integrity and to improve future CBPR endeavors in general, we now elaborate on some of the challenges CREST.BD has encountered in applying CBPR (see also Michalak et al., 2015), and offer some pragmatic solutions. The Delphi team included individuals with lived experience of $\mathrm{BD}$ who had the dedicated time, were in stable health and economic circumstances, and had higher education. Significant mood fluctuations may occur for people with BD involved in research, and may impact study timelines. If anticipated and planned for, teams may, however, lessen the risk of this. For instance, ensuring that responsibilities are shared among several peer researchers, having contingency plans if symptoms do manifest, maintaining open dialogue, and providing supportive supervision can all help reduce delays. Given these accommodations, the benefits of tapping into the knowledge and potential of peer researchers should continue to outweigh any periodic interruptions that may occur. The incorporation of peer researchers exemplifies CREST.BD's commitment to integrating CBPR principles with a Delphi study (Michalak et al., 2016a).

Absent from our team were people situated in more marginalized contexts, for example, from lower socioeconomic positions, racialized identities, and/or from rural or remote settings; this limits the complete understanding of self-management and its application, and the ways to tailor and disseminate findings to more diverse populations. Therefore, developing strategies to access and include people who have historically been, or who continue to be excluded, such as Indigenous Peoples, racialized immigrants, and people with multiple health 
issues will continue to be a strategic focus in our work.

Funding for CBPR projects tends to come from grants that are generally more accessible to people in academic positions, and thus may exclude, or create barriers for people without formal academic standing. Peer researchers who are without academic affiliations or without research experience need, and deserve, appropriate remuneration for sustained participation. We have found it useful to seek out multiple sources of funding, and when preparing grant applications, to budget well for peer researcher remuneration, travel, childcare costs, etc. Further, the emergent design elements of CBPR (i.e., plans may change as decision-making is shared and as learning occurs), may impede initial project goals.

The process of CBPR as a whole draws attention to some challenges that may surface when adopting the approach. Time or lack thereof, may be an issue given that the capacities and research skills of the community may need to be nurtured and developed. Academic researchers may also be new to CBPR and require mentorship and capacity building. Teams can expect the pace of progress to be varied and may need to adjust timelines accordingly. Effective communication and authentic partnerships will also require extended engagement between partners. It is important for partners to gauge the optimal frequency and mode of involvement in order to maintain continuous, but not onerous, terms of engagement.

\section{Conclusions}

As Jorm (2015) summarized, Delphi consensus consultation methods offer an important complement to traditional mental health research methodologies. Here we have described, as of yet, a relatively untapped opportunity; the combination of Delphi consensus consultation methods with CBPR. The complementary integration of these two research methodologies holds potential to meet multiple goals. First, the involvement of key stakeholders in research processes can improve research quality. For example, in our described project, we were able to maximize participant recruitment and retention, and rely on community expertise to nimbly address emergent study issues and concerns. Second, the combination of Delphi consensus consultation methods and CBPR lends itself to improved knowledge exchange. Given that the project's peer researchers produced ongoing process-level outputs (i.e., blogs), which described their experiences and the study, we were able to circumvent the lag in time that is frequently observed when research relies solely on final outputs such as peer-reviewed publications. Third, stakeholder involvement helps build community capacity for engaging in research and in knowledge exchange, which in turn meets a core strategic goal for our CREST.BD network.

In this specific area, future research should investigate, and produce knowledge on effective self-management in partnership with ethnically diverse communities. As noted by Michalak et al. (2012), CBPR methods are best suited to access traditionally hard-to-reach $\mathrm{BD}$ populations and other marginalized communities. Indeed, one of the key mandates for CREST.BD is to develop tailored engagement strategies, appropriate and sensitive training and capacity-building exercises, and continuous evaluations to ensure that the barriers that deter marginalized communities from participating in research are addressed. Taken together, the thoughtful integration of Delphi consensus consultation methods with CBPR represents 
a promising approach for achieving the final and critical goal of Delphi studies - to transform research findings into real world action.

\section{Acknowledgements}

CREST.BD is a Canadian government (Canadian Institutes of Health Research) funded multidisciplinary network dedicated to advancing bipolar disorders research and knowledge exchange, particularly embracing community-based participatory research. Funding provided by Coast Capital Depression Research. With thanks to our project volunteers (Soma Barsen, Cassie Cowie, Tanya Lee and Chanel Wood), Professor Anthony Jorm for project consultation, and our Delphi panel experts.

\section{About the Authors}

Jehannine Austin is a member of CREST.BD, and the Departments of Psychiatry and Medical Genetics at UBC.

Steven J. Barnes is a member of CREST.BD and the Department of Psychology at the University of British Columbia.

Anusha Balram is a trainee with CREST.BD and a PhD student at Ryerson University.

Lesley Berk is a member of CREST.BD and works within the IMPACT Strategic Research Centre, School of Medicine, Faculty of Health, Deakin University, Geelong, Australia and the Department of Psychiatry, University of Melbourne, Victoria, Australia.

Sara Lapsley is a member of CREST.BD and a PhD student at Carleton University.

Sharon Hou is a trainee with CREST.BD and PhD student at the University of Guelph.

Erin E. Michalak (corresponding author) is lead of CREST.BD and the Department of Psychiatry at the University of British Columbia. Email: erin.michalak@ubc.ca

Dragos C. Ragazan is currently with the Department of Psychiatry, Lund University and Child and Adolescent Psychiatry, Region Skåne, Sweden. He was a trainee with CREST.BD at the time of writing of this paper. 
Mike Scott was a peer researcher with CREST.BD when this Delphi project was being conducted.

Melinda J. Suto is a member of CREST.BD and the Department of Occupational Science and Occupational Therapy at the University of British Columbia.

\section{References}

Amos, T., \& Pearse, N. (2008). Pragmatic research design: An illustration of the use of the Delphi technique. Electronic Journal of Business Research Methods, 6(2), 95-102.

Berk, L., Jorm, A. F., Kelly, C. M., Dodd, S., \& Berk, M. (2011). Development of guidelines for caregivers of people with bipolar disorder: A Delphi expert consensus study. Bipolar Disorders, 13(5-6), 556-570.

Chatterton, M. L., Stockings, E., Berk, M., Barendregt, J. J., Carter, R., \& Mihalopoulos, C. (2017). Psychosocial therapies for the adjunctive treatment of bipolar disorder in adults: Network meta-analysis. The British Journal of Psychiatry, 210(5), 333-341.

Davidson, P. L. (2013). The Delphi technique in doctoral research: Considerations and rationale. Review of Higher Education and Self-Learning, 6(22), 53-65.

Donohoe, H., Stellefson, M., \& Tennant, B. (2012). Advantages and limitations of the e-Delphi technique: Implications for health education researchers. American Journal of Health Education, 43(1), 38-46.

Elo, S., \& Kyngäs, H. (2008). The qualitative content analysis process. Journal of Advanced Nursing, 62, 107-115.

Fletcher, A.J., \& Marchildon, G.P. (2014) Using the Delphi method for qualitative, participatory action research in health leadership. International Journal of Qualitative Methods, 13, 1-18.

Freire, P. (1972). Pedagogy of the Oppressed. Harmondsworth, Eng: Penguin Books.

Gordon, T. \& Helmer, O. (1964). RAND Report P-2982 Report on a long-range forecasting study. https://www.rand.org/pubs/papers/P2982.html

Goodwin, F., \& Redfield-Jamison, K. (2007). Manic depressive illness: Bipolar disorders and recurrent depression (2 ed.). New York: Oxford University Press.

Ilieva, S. (2013). Delphic Oracle, RAND corporation and Delphi method. Journal Scientific and Applied Research, 4, 192-199.

Israel, B., Eng, E., Schulz, A., Parker, E., \& Satcher, D. (Eds.). (2005). Methods in community-based participatory research for health. San Francisco: Jossey-Bass.

Israel, B., Schulz, A., Parker, E., \& Becker, A. (1998). Review of community-based research: Assessing partnership approaches to improve public health. Annual Review of Public Health, 19, 173-202.

Jorm, A. F. (2015). Using the Delphi expert consensus method in mental health research. Australian \& New Zealand Journal of Psychiatry, 49(10), 887-897.

Kezar, A., \& Maxey, D. (2016). The Delphi technique: An untapped resource of participatory research. International Journal of Social Research Methodology, 19(2), 143-160. 
Landeta, J., Barrutia, J., \& Lertxundi, A. (2011). Hybrid Delphi: A methodology to facilitate contribution from experts in professional contexts. Technological Forecasting \& Social Change, 78, 1629-1641.

Linstone, H.A. \& Turoff, M. (2011). Delphi: A brief look backward and forward. Technological Forecasting \& Social Change, 78, 1712-1719.

Merikangas, K. R., Akiskal, H. S., Angst, J., Greenberg, P. E., Hirschfeld, R. M. A., Petukhova, M., \& Kessler, R. C. (2007). Lifetime and 12-month prevalence of bipolar spectrum disorder in the National Comorbidity Survey replication. Archives of General Psychiatry, 64(5), 543-552. doi: 10.1001/archpsyc.64.5.543

Michalak, E., Hole, R., Livingston, J., Murray, G., Parikh, S., Lapsley, S., \& McBride, S. (2012). Improving care and wellness in bipolar disorder: Origins, evolution, and future directions of a collaborative knowledge exchange network. International Journal of Mental Health Systems, 6(16).

Michalak, E., Jones, S., Lobban, F., Algorta, G. P., Barnes, S. J., Berk, L., . . CREST.BD. (2016a). Harnessing the potential of community-based participatory research approaches in bipolar disorder. International Journal of Bipolar Disorders, 4(4).

Michalak, E., Lane, K., Hole, R., Barnes, S., Khatri, N., Lapsley, S., . . Johnson, S. (2015). Towards a better future for Canadians with bipolar disorder: Principles and implementation of a community-based participatory research model. Engaged Scholar Journal: Community-Engaged Research, Teaching, and Learning, 1(1), 132-147.

Michalak, E., Suto, M., Barnes, S., Hou, S., Lapsley, S., Scott, M., . . CREST.BD. (2016b). Effective self-management strategies for bipolar disorder: A community-engaged Delphi consensus consultation study. Journal of Affective Disorders, 206, 77-86. doi: 10.1016/j.jad.2016.06.057

Michalak, E., Yatham, L., Kolesar, S., \& Lam, R. (2006). Bipolar disorder and quality of life: A patient-centered perspective. Quality of Life Research, 15(1), 25-37.

Minas, H., \& Jorm, A. F. (2010). Where there is no evidence: Use of expert consensus methods to fill the evidence gap in low-income countries and cultural minorities. International Journal of Mental Health Systems, 4(1), 33.

Minkler, M., \& Wallerstein, N. (2003). Community based participatory research for health. San Francisco: Jossey-Bass.

Nair, R., Aggarwal, R., \& Khanna, D. (2011). Methods of formal consensus in classification/ diagnostic criteria and guideline development. Seminars in Arthritis and Rheumatism, 41(2), 95105.

Powell, C. (2003). The Delphi technique: Myths and realities. Journal of Advanced Nursing, 41(4), 376382.

Rowe, G. \& Wright, G. (1999). The Delphi technique as a forecasting tool: Issues and analysis. International Journal of Forecasting, 15, 353-375.

Rosa, A. R., Reinares, M., Michalak, E. E., Bonnin, C. M., Sole, B., Franco, C., . . Vieta, E. (2010). Functional impairment and disability across mood states in bipolar disorder. Value Health, 13(8), 984-988.

Sackett, D. L., Straus, S. E., Richardson, W. S., Rosenberg, W., \& Haynes, R. B. (2000). Evidence-based medicine: How to practice and teach EBM (2 ed.). Edinburgh: Churchill Livingstone.

Schneider, B. (2012). Participatory action research, mental health service user research, and the hearing (our) voices projects. International Journal of Qualitative Methods, 11(2), 152-165.

Engaged Scholar Journal: Community-Engaged Research, Teaching, and Learning 
Surowiecki, J. (2004). The wisdom of crowds: Why the many are smarter than the few and how collective wisdom shapes business, economies, societies, and nations. New York: Doubleday.

Suto, M., Murray, G., Hale, S., Amari, E., \& Michalak, E. (2010). What works for people with bipolar disorder? Tips from the experts. Journal of Affective Disorders, 124(1-2), 76-84.

Totikidas, V. (2010). Applying the Nominal Group Technique (NGT) in community based action research for health promotion and disease prevention. Australian Community Psychologist, 22 (1), 18-29.

Vázquez-Ramos, R., Leahy, M., \& Estrada Hernández, N. (2007). The Delphi method in rehabilitation counseling research. Rehabilitation Counseling Bulletin, 50(2), 111-118.

Vernon, W. (2009). The Delphi technique: A review. International Journal of Therapy and Rehabilitation, 16(2), 69-76.

Viswanathan, M., Ammerman, A., Eng, E., Garlehner, G., Lohr, K. N., Griffith, D., . . Whitener, L. (2004). Community-based participatory research: Assessing the evidence. Evidence Reports/ Technology Assessment (Summary)(99). Retrieved from https://www.ncbi.nlm.nih.gov/books/ NBK11852/ 